\title{
Documento de postura: la afectación de personal médico y de la salud en la pandemia de SARS-CoV-2
}

\author{
Position paper: Impact on medical and health personnel in the SARS-CoV-2 pandemic \\ Teresita Corona, * Jorge A. Castañón-González, Patricia Clark-Peralta, \\ Carmen García-Peña, Rosalinda Guevara-Guzmán, Guillermo Domínguez-Cherit, José Halabe-Cherem, \\ Malaquías López-Cervantes, Alejandro Macías-Hernández, Mayela Rodríguez-Violante, \\ Patricio Santillán-Doherty, Gilberto Vázquez-de Anda y Alberto Lifshitz-Guinzberg \\ Academia Nacional de Medicina de México, Comité de Análisis de la Pandemia COVID-19, Ciudad de México, México
}

La Academia Nacional de Medicina de México, como organismo académico, educativo, de reflexión, representativo de la medicina mexicana y consultivo del Gobierno Federal, ha estado atenta al desarrollo de la infección por SARS-Cov2 en el país y manifiesta su interés por contribuir a que se logren los mejores desenlaces posibles.

Los médicos, por la esencia de su profesión y al igual que otros trabajadores de la salud, están expuestos naturalmente a los riesgos de contagio que supone el contacto con pacientes infectados, pero parte importante de su profesión es aprender a protegerse de estos peligros y evitar diseminarlos. Aun así, como lo muestra la historia, ocurren casos en los que estas medidas de protección no se aplican óptimamente y las personas resultan afectadas.

La Academia ha sido sensible al constatar que la pandemia de COVID-19 ha afectado en forma considerable a médicos y personal de salud, quienes han tenido en muchos países, incluyendo México, una frecuencia mayor de la esperable de contagios y muertes, aunque no conocemos la cifra exacta. Más de 130 países han reportado muertes en los profesionales de este sector; las cifras de personal infectado en series internacionales varían de 3.5 a $29 \%$ en China, $10.7 \%$ en Italia y 15.6 a $19 \%$ en Estados Unidos. $^{1}$

La afectación del personal médico en esta pandemia indica la existencia de un deber supererogatorio implícito, en otras palabras, la necesidad de actuar más allá de lo que las obligaciones especificadas exigen. El gremio médico tiene un papel ocupacional que van más allá de lo estipulado en contratos o convenios, al estar gobernado por principios éticos que van desde la misma concepción histórica de la relación médico-paciente, pasando por el profesionalismo atado a la virtud de honrar el compromiso de la sanación y terminando con el acuerdo o consentimiento de participar en un pacto social del que derivan poderes y obligaciones que lo sujetan. Uno de ellos es el "deber de tratar", entendido como la asistencia a otros a pesar de los riesgos inherentes. ${ }^{2}$ Los riesgos asumidos por médicas y médicos son evidentes: mientras que la inmensa mayoría de la población se ha sometido a políticas de contención y mitigación, los profesionales de la medicina no solo se han mantenido en el trabajo, sino que, en muchas circunstancias, se enfrentan directamente a pacientes infectados de SARS-CoV-2, con la exposición a aerosoles, áreas e insumos contaminados con el virus. Esta situación ha sido discutida históricamente desde el inicio de la epidemia del virus de la inmunodeficiencia humana y, más recientemente, a raíz de los brotes de los síndromes respiratorio agudo grave, de Oriente Medio y Ébola; la presente pandemia nos somete nuevamente a prueba.

Por otro lado, es un hecho bien conocido el déficit de personal médico en general en México (donde la 
media es de 2.4 médicos por 1000 habitantes, cuando en los países de la Organización para la Cooperación y el Desarrollo Económicos es de 3.5); ${ }^{3}$ esta pandemia ha puesto en evidencia la escasez de personal capaz de manejar el exceso de los casos graves que se presentan (especialistas en cuidados intensivos, neumología, anestesiología, infectología, medicina interna, entre otros).

La supererogación y la escasez sustentan el argumento de que el personal médico debe considerarse como un recurso valioso cuya sustentabilidad debe ser cuidada en extremo.

Un estudio del Instituto Nacional de Ciencias Médicas y Nutrición Salvador Zubirán ${ }^{4}$ muestra que el personal de salud representa $13 \%$ de los casos informados en la Ciudad de México, aunque el número de pruebas realizadas fue mayor que las que se efectuaron en proporción en el resto de la población. Entre los pacientes tratados por esta infección en el mismo Instituto, $18.6 \%$ fue trabajador de la salud. ${ }^{5}$ En otro estudio realizado en el Instituto Nacional de Geriatría ${ }^{6}$ se reporta que el número de pruebas en trabajadores de la salud fue casi 10 veces mayor que en la población general (34 349 versus 3825 por 100 000), con lo que se identificaron más casos asintomáticos y leves entre el personal de salud. Por esa razón, la letalidad en personal de salud fue menor que en la población general, aunque la tasa poblacional de mortalidad fue mayor (195 versus 125 muertes por 100 000). Lo anterior constituye una seria preocupación para esta Academia.

Una política de capacitación del personal, con acceso suficiente y adecuado a los equipos de protección, así como un programa de medicina ocupacional que incluya la realización liberal y amplia (en personal sintomático y sus contactos asintomáticos) de pruebas de PCR para SARS-CoV-2 pueden ayudar a mantener una prevalencia reducida de COVID-19 de origen nosocomial en el personal de salud., Una parte de los trabajadores de la salud ha adquirido la infección en la comunidad (en el Instituto Nacional de Enfermedades Respiratorias, 128 de 3481 tuvieron infección nosocomial, sin ninguna defunción, y 231 de 3481 la adquirieron en la comunidad, con dos defunciones [datos no publicados]).

Consideramos que los cuidados para evitar los contagios no debieran aplicarse solo cuando los médicos y el personal de salud se exponen a pacientes diagnosticados con COVID-19; en situaciones de epidemia deberían emplearse ante todos los enfermos, porque entre ellos puede haber algunos con infección asintomática, igualmente transmisible. Además, la capacitación tiene que ser reforzada, preferentemente mediante actividades presenciales antes de que el personal de salud se ponga en contacto con personas infectadas de SARS-CoV-2 o con COVID-19, en particular en el personal de reciente contratación y en quienes ha sido reasignados exprofeso para la atención de pacientes con COVID-19, tanto en áreas de hospitalización como de consulta externa. Por lo general, este personal, al no estar familiarizado con la atención de pacientes graves con SARS-CoV-2, es vulnerable a la infección.

Por tal motivo, la Academia expresa su recomendación de utilizar tales medidas ante todos los pacientes y extremar los cuidados reconocidos, como que el personal de salud de las áreas designadas como COVID cuente en todo momento con el equipo de protección personal necesario: mascarilla, cubrebocas o máscara de alta eficiencia (N95, NK95 o equivalentes o superiores), así como de protección ocular y equipos adicionales como batas y guantes, que determinen las políticas de la institución. Todo el personal de salud que tenga contacto con pacientes, incluyendo quienes trabajan en áreas no designadas como COVID, debería tener acceso a mascarillas o cubrebocas quirúrgicos de triple capa o de eficacia superior y al equipo adicional que determine la institución, así como a planes y políticas locales para que las áreas de trabajo se mantengan ventiladas y para evitar que se saturen.

\section{Medidas para evitar el contagio del personal de salud}

- De acuerdo con las actividades que espera realizar, el personal médico debe recibir capacitación específica en las medidas de protección necesarias.

- Uso todo el tiempo de mascarillas o cubrebocas de triple capa, con moldeador nasal.

- Protección ocular con lentes industriales o careta.

- Procurar que los trabajadores de salud que atienden COVID no trabajen en distintos hospitales, para lo cual será conveniente mejorar su percepción económica.

- Realizar muestreo de casos con base en los síntomas de sospecha y el rastreo de contactos.

- Continuar con la atención especial a trabajadores con multimorbilidad y en condiciones de vulnerabilidad, para identificar la infección en forma oportuna y garantizar el tratamiento. 
La mejor estrategia para proteger a los trabajadores de la salud es reducir la circulación comunitaria de SARS-Co-2. Para ello, es fundamental la participación de la sociedad, manteniendo las medidas del uso universal del cubrebocas y el distanciamiento físico, por más desgaste que esto implique.

\section{Bibliografía}

1. Bandyopadhyay S, Baticulon RE, Kadhum M, Alser M, Ojuka DK, Badereddin $Y$. Infection and mortality of healthcare workers worldwide from COVID-19: a scoping review. medRxiv [Preprint]. 2020 Jun 05. DOI: 10.17605/OSF.IO/JYUXB

2. Daniels N. Just health: meeting health needs fairly. Reino Unido: Cambridge University Health; 2008.
3. Organisation for Economic Co-operation and Development [Internet]. Francia: Health at a Glance 2019; 2019.

4. Guerrero-Torres L, Caro-Vega Y, Crabtree-Ramírez B, Sierra-Madero JG. Clinical characteristics and mortality of healthcare workers with SARS-CoV-2 infection in Mexico City. Clin Infect Dis. 2020.

5. Ortiz-Birzuela E, Villanueva-Reza M, González-Lara MF, Tamez-Torres KM, Román-Montes CM, Díaz-Mejía BA, et al. Clinical and epidemiological characteristics of patients diagnosed with COVID-19 in a tertiary care center in Mexico City: a prospective cohort study. Rev Invest Clin. 2020;72:165-177.

6. Antonio-Villa NE, Bello-Chavolla OY, Vargas-Vázquez A, Fermín-Merínez C, Márquez-Salinas A, Pisanty-Alatorre J, et al. Assessing the burden of COVID-19 amongst healthcare workers in Mexico City: A data-driven call to action. Clin Infect Dis. 2020.

7. Ñamendy-Silva SA. Health care workers on the frontlines of COVID-19 in Mexico. Heart Lung. 2020;29:1.

8. Kaur R, Weiss TT, Perez A, Fink JB, Chen R, Luo F, et al. Practical strategies to reduce nosocomial transmission to healthcare professionals providing respiratory care to patients with COVID-19. Critical Care. 2020;24:571. 